UNIVERSIDAD SANTO TOMAS DE AQUINO

LA CONFIANZA ENTRE DIRECTIVOS-COLABORADORES Y SU INFLUENCIA EN LAS FINANZAS

CRISTIAN ALEXIS SANTACRUZ APONTE

ESTUDIANTE ADMINISTRACION DE EMPRESAS

CODIGO: 2190902 


\section{La confianza entre directivos-colaboradores y su influencia en las finanzas}

\section{Resumen}

Durante años muchas empresas se han preguntado cómo crear una verdadera confianza entre sus directivos y colaboradores, que beneficie ambas partes y lleve a las organizaciones a alcanzar los primeros lugares en sus negocios como resultado de dicha relación. Pero esta relación directivo-colaborador, tiene muchos aspectos que se deben tener en cuenta para entender como diferentes características de los seres humanos influencian en los resultados de la organización.

La generación de confianza, puede depender de factores que rodean la relación directivo-colaborador, como clima organizacional y liderazgo, competencias y virtudes directivas, inteligencia emocional, y la confianza en sí misma. Las cuales se analizaran para comprender como estas variables influencian dicha relación.

Las finanzas son influenciadas por la confianza, pues son el reflejo del trabajo entre directivos-colaboradores, y si estos dos mejoran su relación esto se verá reflejado en las ganancias de una empresa, ya que toda organización debe primero funcionar internamente y luego externamente con los clientes, Richard Brandson creador de virgin group, uno de los grupos de empresas más prestigiosos del mundo, dijo en una entrevista que si cuidas de tus colaboradores, ellos cuidaran a tus clientes (Cárdenas, 2015).

\footnotetext{
Abstract

For years many companies have wondered how to create a true trust between their managers and collaborators, which benefits both parties and leads organizations to reach the top places in their business as a result of this relationship. But this directivecollaborative relationship has many aspects that must be taken into account to
} 
understand how different characteristics of human beings influence the results of the organization. The generation of trust may depend on factors surrounding the managerial-collaborative relationship, such as organizational climate and leadership, competencies and managerial virtues, emotional intelligence, and self-confidence. Which will be analyzed to understand how these variables influence this employment relationship.

The finances are influenced by trust, as they are the reflection of the work between managers-collaborators, and if these two improve their relationship this will be reflected in the profits of a company, since every organization must first function internally and then externally with the clients, Richard Brandson, creator of the virgin group, one of the most prestigious groups of companies in the world, said in an interview that if you take care of your employees, they will take care of your clients (Cárdenas, 2015).

\section{Palabras Clave}

Confianza, liderazgo, finanzas, relaciones y competencias.

\section{Key Words}

Trust, leadership, finance, relationships and competencies.

\section{Objetivo general}

Entender que las ganancias o pérdidas de una organización no son solo influenciadas por temas meramente financieros sino que aspectos como la confianza juegan un papel importante en los resultados financieros.

\section{Objetivos específicos}

1. Abarcar como la confianza entre directivos-colaboradores influencia las ganancias o pérdidas organizacionales. 
2. Entender como distintas variables afectan la confianza entre directivoscolaboradores.

3. Comprender que la confianza es un aspecto importante para las organizaciones.

\section{Introducción}

Los países en desarrollo tienen muchas diferencias con los países que son potencia mundial, y una de las principales diferencias son las finanzas. Estas diferencias tienen unos aspectos principales entre los países en vía de desarrollo y los desarrollados en cuestiones de finanzas que no solo se centran en los mercados internacionales, sino que analizando cuestiones internas las principales diferencias pueden ser las siguientes:

La incapacidad de los países para tener sus deudas externas con sus propias monedas locales hace que dependan del dólar y este puede aumentar o disminuir severamente sus deudas, las brechas en el desarrollo de los sistemas financieros y los mercados capitales que converge en una falta de instrumentos financieros en el largo plazo, los mercados internos muchas veces son muy pequeños comparados con los de otros países, etc. (García, 2008).

La desigualdad financiera de las personas también es un aspecto importante entre los países, por ejemplo en los Estados Unidos la desigualdad entre los años 2003 y 2007 fue que el aumento del salario para los directores generales fue del 45 por ciento, para los ejecutivos del 15 por ciento, y para los demás colaboradores de menos del 13 por ciento (Bollé, 2008). Y esos porcenteajes podrían ser mayores en los países en desarrollo.

Pero no solo las situaciones de un país afectan a las organizaciones en cuestiones financieras, sino que también los aspectos internos de cada empresa se verán reflejados dentro de los resultados financieros. Por esto es importante revisar qué aspectos son los que más afectan a una organización, y el principal aspecto puede ser el recurso humano, 
ya que son las personas las que están detrás de las empresas, son ellas las que las impulsan o las detienen, son los integrantes de las organizaciones los que mueven todas las áreas posibles de una empresa ya sea directa o indirectamente pues hay automatizaciones pero de nada sirven si no hay personas que sepan encaminar esas automatizaciones hacia el desarrollo empresarial, el cual se verá reflejado en las ganancias o pérdidas de cada periodo.

Y si analizamos este recurso humano, una las principales características que se debe potencializar es la confianza entre los directivos-colaboradores, la cual no solo mejorara las finanzas de una empresa sino que también mejorara muchos otros aspectos como el liderazgo, clima organizacional, motivación, etc.

\section{Área de Talento Humano}

El área de talento humano tiene un rol importante en esta relación directivocolaborador. Díaz, Cardona y Aguirre (2014) afirman: "El área de recursos humanos (RRHH) asume responsabilidades fundamentales para el desarrollo de la empresa,... Es allí donde se desarrollan las relaciones internas y externas que buscan mantener la estructura de relaciones que sostiene la organización.” (p. 2). Por esto, es importante que cada empresa posea un equipo de recursos humanos ideal para su organización, y para esto debe entender que (RRHH) no es un área funcional, sino que es tan estratégica como pueden ser las áreas de finanzas, contaduría, planeación, etc, por lo cual talento humano deberá procurar el ambiente propicio para la generación de confianza.

La conciencia y la responsabilidad son percibidas como las mejores señales para tomar una decisión basada en la confianza, pues transmiten esa seguridad (Hu y Wang, 2014). Es muy importante lo que las personas a nuestro alrededor perciben de nosotros para 
generar una confianza en lo que hacemos, pues todos tendemos analizar a los demás, pues sus gestos nos pueden transmitir confianza o inseguridad.

Y estos aspectos tan pequeños como los gestos son importantes para la generación de confianza, la cual está implícita de una forma cualitativa en las finanzas de una organización.

Pero hay más variables que ayudaran a una persona a generar confianza, como pueden ser inteligencia emocional, liderazgo, clima organizacional, competencias y virtudes, entender los géneros masculino y femenino, que pueden ayudar a entender como la generación de confianza depende de muchos aspectos, que afectan a una organización.

\section{Clima Organizacional y Liderazgo}

Las características del clima organizacional pueden ser percibidas por los colaboradores de forma directa e indirecta (Serrano y Portalanza, 2014). Por esto cada trabajador puede tener una percepción distinta de sus compañeros del ambiente laboral de una empresa, pues para uno será positivo y para otro negativo dicho ambiente. Por ejemplo, cuando una persona percibe un ambiente de estrés y este llega a ser agudo, será más difícil la generación de confianza (Potts, McCuddy, Jayan y Porcelli, 2019).

Díaz, Cardona y Aguirre (2014) hablan de la cultura organizacional como: "Un patrón complejo de creencias, expectativas, ideas, valores, actitudes y conductas compartidas por los integrantes de una organización”. (p. 2). Estas características de la cultura organizacional influencian el clima laboral, y pueden crear un ambiente propicio para una adecuada generación de la confianza, pues si son bien potencializadas por una empresa se podrían ver como la plataforma para que cada directivo-colaborador crezca en confianza y esto se reflejara en ganancias monetarias a futuro. 
El clima ético organizacional también es un aspecto importante, en un estudio realizado por Nedkovski, Guerci, De Battisti y Siletti (2017) se evidencia como la confianza se relaciona con los trabajadores, sus tres resultados más importantes son:

"H1: Un clima ético benevolente se asocia positivamente con la confianza en los colegas, la confianza en el supervisor y la confianza en la organización.

H2: Un clima ético basado en principios se asocia positivamente con la confianza en los colegas, la confianza en el supervisor y la confianza en la organización.

H3: Un clima ético egoísta se asocia negativamente con la confianza en los colegas, la confianza en el supervisor y la confianza en la organización.”

Por estola ética es importante para generar un ambiente donde la confianza pueda florecer.

El liderazgo es una de las principales variables que influencian el clima organizacional, y que termina repercutiendo en la generación de confianza. Un líder puede generar la calidad del clima organizacional, debido a la percepción que se les trasmite a los colaboradores, también un líder es el encargado de influir en los trabajadores de forma positiva, esto con el fin de generar una motivación que los lleve a una excelencia organizacional y que generara ganancias a una organización primero de forma cualitativa y luego de forma cuantitativa.

Un aspecto fundamental para el líder son sus prácticas, ya que estas pueden mejorar el desempeño organizacional. También debe fomentar el crecimiento personal, desarrollar habilidades y competencias de todos sus colaboradores. (Serrano y Portalanza, 2014).

Por eso el líder, debe buscar un liderazgo que esté alineado a la productividad organizacional teniendo en cuenta las relaciones interpersonales dentro de unos 
parámetros de liderazgo que incluyan el apoyo y la confianza mutua con todos los colaboradores.

En un estudio en el país Camerún, donde la situación económica puede ser complicada para emprender y desarrollar empresas, se realizó un estudio para resaltar las características de los empresarios que han tenido éxito con sus empresas en un ambiente tan complejo, el estudio fue realizado en PYMES. Los factores que debe tener todo directivo para sobrevivir en un contexto empresarial como el de Camerún, son; el deseo de autonomía, autoestima, ética del líder, altruismo, sentimiento personal de logro y el deseo de independencia. Todos relacionados según el mismo estudio con la confianza (Boubakary, 2015). Estas características las debería tener cada líder, ya que al poseerlas también generara confianza en las personas que tiene alrededor.

El liderazgo de las mujeres, suele ser más democrático y ellas también son más sensibles al contexto grupo cuando hay decisiones que afectan a todos (Ertac y Gurdal, 2019). Por lo cual pueden tener una generación de confianza mayor al delegar decisiones.

\section{Competencias y virtudes}

La teoría de la psicología positiva, puede incluir características como; Sabiduría y conocimiento, coraje, justicia, templanza, transcendencia y humildad (Serrano, 2017). El propósito de esta psicología positiva es hacer que la persona llegue a su máximo potencial, por medio de su carácter. Estas características de la psicología positiva las deben tener en cuenta todos los directivos-colaboradores ya que están de alguna forma relacionadas con la generación de confianza, la falta de alguna de las características mencionadas puede desequilibrar las relaciones laborares. La psicología positiva es una muy buena propuesta que se debe tener en cuenta a la hora de generar confianza, pues 
no solo incluye competencias sino también virtudes las cuales hablan de cada persona no solo desde sus habilidades sino también desde su personalidad, pues no se puede pensar que un directivo o colaborador tenga una vida laboral totalmente separada de su vida personal.

Si una empresa analiza cada una de estas características de la psicología positiva, podrá encontrar que fortalezas y debilidades tiene en cuanto a competencias y virtudes, pues al analizarlas en cada individuo, podrá mejorarlas o desarrollarlas para que se vean reflejadas en su organización a manera de ganancias, reputación, trabajo en equipo, etc.

La personalidad de cada persona es distinta, tanto que puede llegar a ser diferente si una persona trabaja sola, con un compañero, o lo hace con un grupo de personas, pues estos tres niveles hacen que una persona actué de forma distinta según cada ocasión (Revelle y Condon, 2015). Esto hace que una persona muestre sus virtudes en cada nivel, y para unos será mejor algún nivel que los otros. Cada directivo debe entender que sus colaboradores sienten más confianza en alguno de los tres niveles (Individual, Con un compañero, y en Grupo), y deberá el directivo encontrar donde el trabajador se desenvuelve mejor, para así ganar confianza con él en la asignación de tareas y crear una mejor posibilidad de ganancias financieras en los trabajos realizados.

\section{Inteligencia Emocional}

Goleman 1999 (Como se citó en Duque, García y Hurtado 2017) dijo que "La inteligencia emocional (IE) se define como la capacidad del individuo de reconocer los sentimientos propios y ajenos, motivarse y manejar sus emociones de manera adecuada, y los efectos en su comportamiento y en la relación con los demás.”

Las competencias laborales e individuales tienen una influencia directa por parte de la inteligencia emocional, ya que las competencias vienen de las conductas y actitudes, las 
cuales son afectadas directamente por las emociones y los sentimientos (Duque, García y Hurtado, 2017). Pero se debe tener en cuenta que la inteligencia emocional en si no desarrolla ninguna capacidad sino que es una variable que es relevante para desarrollar las capacidades laborales. Y esta inteligencia emocional sirve para situaciones con los clientes, como por ejemplo en el área de ventas, el trato que recibirá el cliente requiere que cada colaborador tenga una inteligencia emocional suficiente para brindar el mejor servicio posible, ya que se pueden presentar casos donde los clientes sean groseros, toscos y hasta irrespetuosos, y cada colaborador deberá persuadir de la mejor manera al cliente para que realice la compra, tratando de cambiar su actitud frente a los bienes y servicios que ofrecerá de la empresa, de esta forma generan una mayor ganancia para la empresa. Pero estos tratos por parte de los clientes son mal vistos aunque la fuerza de ventas sea muy amable, y es posible que los colaboradores puedan soportar de una forma distinta a los clientes que a sus jefes, pues saben que lo que esperan del cliente principalmente es su compra, pero de sus jefes pueden esperar un mejor trato, es aquí donde el contrato psicológico entra en acción.

El contrato psicológico, está inmerso en cada relación laboral, aunque no se firma físicamente, está basado en las promesas que perciben las personas acerca de una organización (Guaderrama, Cavazos y Reyes, 2017). Este contrato no solo implica las promesas recibidas por escrito en los contratos laborales, sino también las promesas o expectativas que cada persona se crea de su organización, este contrato es relevante para entender como las emociones se relacionan con cada puesto de trabajo, pues este contrato es muy subjetivo. Esto nos ayuda a entender que cada persona percibirá su trabajo de forma distinta y así mismo estará motivada en su día a día. Este contrato, implica analizar el agotamiento emocional. 
El agotamiento emocional hace que las personas no solo se sientan casadas mentalmente sino también físicamente (Guaderrama, Cavazos y Reyes, 2017). Por eso es importante que los directivos entiendan que si quieren generar confianza con sus colaboradores, no solo se deben relacionar con ellos desde el punto de vista laboral, sin importarles que situaciones personales están sucediendo con cada colaborador, pues esas situaciones personales pueden generar agotamiento emocional. Pero también este agotamiento emocional se da cuando no hay una buena relación entre directivos-colaboradores porque cada parte llegara a su puesto de trabajo con estrés, frustración, decepción, etc. Y es ahí donde las promesas organizacionales del contrato psicológico se pueden frustrar al no cumplir con las expectativas intrínsecas aunque si estén cumpliendo con las escritas, que pueden ser dinero, bonos, tiempos de trabajo, etc.

\section{Diferencias de Género}

Una de las sugerencias que explica la brecha de géneros en las empresas, es que los hombres y las mujeres no tienen las mismas inclinaciones hacia las negociaciones, pues se pueden comportar de formas distintas (Säve-Söderbergh, 2019).

Un aspecto importante es el salario, pues por ejemplo, acá se puede evidenciar que existen diferencias entre hombres y mujeres. En un estudio que se realizó por SäveSöderbergh sobre las diferencias de género, se encontró que las mujeres piden aumentos de salario menores que los hombres y también ellas obtuvieron una oferta de menor valor que los hombres cuando hicieron dicha petición. (Säve-Söderbergh, 2019).

Los estereotipos de género hacen que se perciba que las mujeres son más cooperativas (piden menos salario) y que los hombres más competitivos (piden más salario) y esto puede ser un punto de vista importante (Säve-Söderbergh, 2019). 
Khalife y Chalouhi, realizaron un estudio sobre las diferencias de género en empresas pyme libanesas, con el fin de encontrar si el género tiene que ver con los ingresos de las empresas. Se demuestra que las mujeres propietarias pueden tener el mismo ingreso en sus empresas que los hombres propietarios, pero las mujeres tienden a asignarse salarios más altos que los de los hombres, pues las mujeres tienen a gastar más dinero en cosas relacionadas con el hogar que los hombres. Es por eso que las empresas de los hombres según muestra el estudio tienen más ingresos brutos que las empresas de las mujeres (Khalife y Chalouhi, 2013).

Se dice que por cuestión de personalidades, en un ambiente de competencia, los hombres se destacan más que las mujeres debido a la competencia misma y más si esta incluye incentivos (Cotton, Li, McIntyre y Price, 2015).

En un juego de negocios desarrollado por la escuela de negocios ESSEC, donde se conformaron equipos de 5 personas al azar, se arrojaron resultados donde se evidencia que los equipos conformados por solo mujeres o solo hombres, no son mejores en ningún momento que los equipos conformados por ambos, tanto hombres como mujeres los equipos mixtos (Lamiraud y Vranceanu, 2018). Esto muestra que aunque se considere a los hombres como más competitivos, nunca tendrán el mismo rendimiento cuando se asocien con mujeres, pues se podría decir que los dos se complementan.

Las mujeres son más competitivas entre ellas mismas que los hombres y también prefieren competir por un cargo con otra mujer que con un hombre (Carpenter, Frank y Vaughn., 2018). Por lo cual el ambiente competitivo de una mujer tiende a aumentar con otra mujer más que con un hombre, pero debemos tener en cuenta como más arriba se dijo, que el mejor equipo laboral no son solo hombres o solo mujeres, sino la unión de los dos. 
Según un estudio realizado en la China, aunque pareciera que las mujeres son menos productivas que los hombres, esto no es cierto, pues se tendría que analizar a qué tipo de trabajo se refiere, pues en los trabajos donde se requiere estudio no hay ninguna diferencia en la productividad de las mujeres y de los hombres (Tsou y Yang, 2019).

Todo lo anterior es importante para entender que la generación de confianza para hombres y mujeres puede ser diferente, dependiendo que cual sea el género al que se quiera acercar como directivo, se deben tener en cuenta los aspectos mencionados ya que la forma de interactuar de cada género es distinta. Y hoy en día con las brechas de género, la confianza entre directivos-colaboradores puede variar de acuerdo al contexto, pero siempre se debe buscar generar confianza tanto en hombres como en mujeres y para eso se debe tener en cuenta beneficios enfocados a cada género.

Hay que resaltar, que a las mujeres se les considera que cuando son directivas, ellas pagan un salario más alto y tienden a imponer sanciones menos severas que los hombres (Chaudhuri, Cruickshank, y Sbai, 2015). Lo cual es un punto a favor muy importante para la generación de confianza hacia los colaboradores.

\section{Confianza}

Hablando de empresas familiares la confianza tiene un impacto más positivo cuando de por medio hay un compromiso (Allen, George y Davis, 2018). La confianza conduce a la cooperación y también es un componente fundamental para que las empresas familiares trabajen juntos con éxito (Kudlats, McDowell y Mahto, 2019).

Comúnmente, se asume que la confianza y el control son opuestos, pero se pudo demostrar que la confianza-control pueden ser sustitutos y/o complementos pues ambos escenarios se pueden desarrollar en una organización (Alpenberg y Scarbrough, 2018). Por lo cual el control puede potencializar la confianza en una organización, pues si se 
sabe ejercer control de una forma en la que las personas sientan seguridad se podrá generar confianza, pero si el control se convierte en una herramienta enfocada hacia el castigo es posible que destruya la confianza.

Las metas trazadas son compromisos, los cuales hacen que las personas tengan un mayor esfuerzo del que tuvieran sin metas, y los incentivos también son considerados como metas, y pueden hacer que haya un mayor rendimiento en un equipo de trabajo (Aguinaga, Cassar, Graham, Skora y Wydick, 2019). Estos incentivos hacen que las personas tengan una confianza que se ve forzada a desarrollarse.

El estado de felicidad en una persona, hace que tienda a otorgar mayor confianza, aun a los desconocidos, y los incentivos en una situación organizacional impulsan la confianza también, pero las emociones positivas son gratis en cambio los incentivos no, por esto se deben utilizar las emociones positivas para desarrollar la confianza pues estas ayudan a que se potencialice. (Mislin, Williams y Shaughnessy, 2015). Y se debe tener cuidado pues es posible que la confianza se rompa cuando no hay emociones positivas que mantengan una motivación que genere confianza.

Cuando hay una interrupción de la confianza, entre dos personas, el hecho de que un tercero ayude a que se recupere la confianza puede ayudar a que la relación se restaure más rápido, pues muchas veces las personas son más abiertas a escuchar a alguien que no las afecto directamente sobre un tema (Yu, Yang y Jing, 2017).

Hay tres caminos cuando una organización rompe la confianza, uno es dar disculpas, otro es presentar excusas y el otro es rechazar la culpa, y cada opción tendrá sus consecuencias negativas o positivas, y luego de escoger alguno de los tres caminos, la manera de conciliarse está basada en que la persona u organización se le debe percibir 
responsabilidad, la cual conduce a la credibilidad percibida y esta última conduce a la reparación de la confianza (Brühl, Basel y Kury, 2018).

Es importante tener en cuenta los escándalos, o los hechos que se conocen de una empresa en su alrededor, ya que los rumores o escándalos pueden afectar la confianza de los colaboradores en una organización, por esto es importante restaurar la confianza o mantenerla en el exterior de la empresa, esta confianza es básicamente la percepción de una empresa desde el público (Bozic, Siebert y Martin, 2019). Una de las formas de restaurar esa confianza hacer que los directivos participen más en las decisiones.

La participación de los colaboradores en las decisiones de los directivos, fomenta las capacidades dinámicas de los trabajadores, y adicionalmente tiene resultados muy positivos para la organización (Wohlgemuth, Wenzel, Berger y Eisend, 2019). Por eso es muy importante que los directivos den participación a colaboradores en reuniones de las empresas, pues esto potencializara a cada trabajador en sus habilidades para que sean dinámicas y también mejorara las decisiones de cada organización.

\section{Conclusión}

Se pudo evidenciar que la confianza está relacionada con otros aspectos que rodean a los trabajadores, y que esos aspectos son muy importantes en el rendimiento del recurso humano de la organización, el cual es el más importante generador de ganancias de las empresas, pues los estados financieros presentados cada periodo son el reflejo del trabajo de cada persona que compone a una organización, cada persona es una tuerca dentro de todo el engranaje organizacional.

Se puede entender entonces que las finanzas no dependen solo de aspectos como el cambio de moneda, el mercado, la inflación, los sistemas financieros, etc. Sino también de otros aspectos como la confianza, la cual es una de las principales generadoras de 
ganancias por medio de su influencia en el rendimiento de cada colaborador. La confianza juega un papel muy importante dentro de cada organización, y resulta relevante estudiar cada aspecto de ella, pues cada variable que influencia la confianza entre directivo-colaborador como se pudo notar más arriba, tienen también su propio campo de estudio porque son aspectos clave dentro del contexto organizacional y tienen características únicas que se pueden encaminar hacia el progreso de las personas y la organización.

\section{Referencias:}

Aguinaga, P., Cassar, A., Graham, J., Skora, L., y Wydick, B., (2019). Raising achievement among microentrepreneurs: An experimental test of goals, incentives, and support groups in Medellin, Colombia. Journal of Economic Behavior \& Organization. Volume (161), 79-97. DOI: https://doi.org/10.1016/j.jebo.2019.02.017

Alpenberg, J., y Scarbrough, D. P., (2018). Trust and control in changing production environments. Journal of Business Research, Volume (88), 527-534. DOI: https://doi.org/10.1016/j.jbusres.2017.12.003

Allen, M. E., George, B. A., y Davis, J. H., (2018). A model for the role of trust in firm level performance: The case of family businesses. Journal of Business Research, Volume (84), 34-45. DOI: https://doi.org/10.1016/j.jbusres.2017.10.048

Bollé, P., (2008). Desigualdad y globalización financiera, en un informe que llega justo a tiempo. Revista Internacional del Trabajo, Vol. 127 Issue 4, p481-487. DOI: https://doi.org/10.1111/j.1564-9148.2008.00044.x 
Boubakary., (2015). The influence of the personal values of the leader on the growth of SMEs in Cameroon. International Strategic Management Review, Volume (3), Issues 1-2, 15-23. DOI: https://doi.org/10.1016/j.ism.2015.09.002

Bozic, B., Siebert, S., y Martin, G., (2019). A strategic action fields perspective on organizational trust repair. European Management Journal, Volume (37), Issue 1, 58-66. DOI: https://doi.org/10.1016/j.emj.2018.04.005

Brühl, R., Basel, J. S., y Kury, M. F., (2018). Communication after an integrity-based trust violation: How organizational account giving affects trust. European Management Journal, Volume (36), Issue 2, 161-170. DOI: https://doi.org/10.1016/j.emj.2017.08.001

Cárdenas, L. F., (2015). Si cuidas a tus empleados, ellos cuidarán de los clientes. Recuperado de https://www.roastbrief.com.mx/2015/10/cuidas-tus-empleadoscuidaran-los-clientes/

Carpenter, J., Frank, R., y Vaughn., E. H., (2018). Gender differences in interpersonal and intrapersonal competitive behavior. Journal of Behavioral and Experimental Economics. Volume (77), 170-176. DOI: https://doi.org/10.1016/j.socec.2018.10.003

Cotton, C., Li, C., McIntyre, F., y Price, J., (2015). Which explanations for gender differences in competition are consistent with a simple theoretical model? Journal of Behavioral and Experimental Economics, Volume (59), 56-67. DOI: https://doi.org/10.1016/j.socec.2015.09.005

Chaudhuri, A., Cruickshank, A., y Sbai, E., (2015). Gender differences in personnel management: Some experimental evidence. Journal of Behavioral and 
Experimental Economics, Volume (58), 20-32. DOI:

https://doi.org/10.1016/j.socec.2015.06.003

Díaz, O., Cardona, M., y Aguirre, D., (2014). Aportes teórico-conceptuales a la dinámica de la gerencia desde el área de gestión humana. Suma Negocios, Volumen (5), Issue 10, 22-28. DOI: https://doi.org/10.1016/S2215$910 X(14) 70005-9$

Duque, J. L., García, M., y Hurtado, A., (2017). Influencia de la inteligencia emocional sobre las competencias laborales: un estudio empírico con empleados del nivel administrativo. Estudios Gerenciales, Volumen (33), Issue 144, 250-260. DOI: https://doi.org/10.1016/j.estger.2017.06.005

Ertac, S., y Gurdal, M. Y., (2019). Preference Communication and leadership in group decision-making. Journal of Behavioral and Experimental Economics. Volume (80), 130-140. DOI: https://doi.org/10.1016/j.socec.2019.03.004

Garcia, J., (2008). Finanzas internacionales y desarrollo: ¿oportunidad o amenaza?. Revista de la Cepal, Issue 96, 157-172. DOI: https://doi.org/10.18356/c3a8fcf5es

Guaderrama, A. I., Cavazos, J., y Reyes, G., (2017) Efecto de la violación del contrato psicológico y el agotamiento emocional sobre el cinismo del empleado. Estudios Gerenciales, Volumen (33), Issue 143, 124-131. DOI: https://doi.org/10.1016/j.estger.2017.04.002

Hu, Z., y Wang, X. T., (2014). Trust or not: Heuristics for making trust-based choices in HR management. Journal of Business Research, Volume (67), Issue 8, 1710171. DOI: https://doi.org/10.1016/j.jbusres.2014.02.017 
Khalife, D., y Chalouhi, A., (2013). Gender and business performance. International Strategic Management Review, Volume (1), Issues 1-2, 1-10. DOI: https://doi.org/10.1016/j.ism.2013.08.001.

Kudlats, J., McDowell, W. C., y Mahto, R. V., (2019). Unrelated but together: Trust and intergroup relations in multi-family businesses. Journal of Business Research. 750-756. DOI: https://doi.org/10.1016/j.jbusres.2018.12.073

Lamiraud, K., y Vranceanu, R., (2018). Group gender composition and economic decision-making: Evidence from the Kallystéebusiness game. Journal of Economic Behavior \& Organization. Volume (145), 294-305. DOI: https://doi.org/10.1016/j.jebo.2017.09.020

Mislin, A., Williams, L. V., y Shaughnessy, B. A., (2015). Motivating trust: Can mood and incentives increase interpersonal trust? Journal of Behavioral and Experimental Economics, Volume (58), 11-19. DOI: https://doi.org/10.1016/j.socec.2015.06.001

Nedkovski, V., Guerci, M., De Battisti, F., y Siletti, E., (2017). Organizational ethical climates and employee's trust in colleagues, the supervisor, and the organization. Journal of Business Research, Volume (71), 19-26. DOI: https://doi.org/10.1016/j.jbusres.2016.11.004

Potts, S., McCuddy, W., Jayan, D., y Porcelli, A., (2019). To trust, or not to trust? Individual differences in physiological reactivity predict trust under acute stress. Psychoneuroendocrinology, Volume (100), 75-84. DOI: https://doi.org/10.1016/j.psyneuen.2018.09.019 
Revelle, W., Condon, D. M., (2015). A model for personality at three levels. Journal of Research in Personality, Volume (56), 70-81. DOI: https://doi.org/10.1016/j.jrp.2014.12.006

Säve-Söderbergh, J., (2019). Gender gaps in salary negotiations: Salary requests and starting salaries in the field. Journal of Economic Behavior \& Organization Volume (161), 35-51. DOI: https://doi.org/10.1016/j.jebo.2019.01.019

Serrano Orellana, B. J., y Portalanza, A. (2014). Influencia del liderazgo sobre el clima organizacional. Suma de Negocios, Volumen (5), Issue 11, 117-125. DOI: https://doi.org/10.1016/S2215-910X(14)70026-6

Serrano, G., (2017). Competencias directivas y virtudes: un camino a la excelencia. Estudios Gerenciales, Volumen (33), Issue 143, 208-216. DOI: https://doi.org/10.1016/j.estger.2017.03.004

Tsou, M. W., y Yang, C. H., (2019). Does gender structure affect firm productivity? Evidence from China. China Economic Review Volume (55), 19-36. DOI: https://doi.org/10.1016/j.chieco.2019.03.005

Wohlgemuth, V., Wenzel, M., Berger, E. S., y Eisend, M., (2019). Dynamic capabilities and employee participation: The role of trust and informal control. European Management Journal, Available online 2. 1-12. DOI: https://doi.org/10.1016/j.emj.2019.02.005

Yu, Y., Yang, Y., y Jing, F., (2017). The role of the third party in trust repair process. Journal of Business Research, Volume (78), 233-241. DOI: https://doi.org/10.1016/j.jbusres.2017.01.015 